\title{
Wash-In Leptogenesis
}

\author{
Valerie Domcke $\odot,{ }^{1,2, *}$ Kohei Kamada $\odot,{ }^{3, \dagger}$ Kyohei Mukaida $\odot,{ }^{1,4, *}$ Kai Schmitz ${ }^{1,},{ }^{1,}$ and Masaki Yamada $\odot^{5,6, \|}$ \\ ${ }^{1}$ Theoretical Physics Department, CERN, 1211 Geneva 23, Switzerland \\ ${ }^{2}$ Institute of Physics, Laboratory for Particle Physics and Cosmology, EPFL, 1015 Lausanne, Switzerland \\ ${ }^{3}$ Research Center for the Early Universe, The University of Tokyo, Hongo 7-3-1 Bunkyo-ku, Tokyo 113-0033, Japan \\ ${ }^{4}$ Deutsches Elektronen-Synchrotron DESY, 22607 Hamburg, Germany \\ ${ }^{5}$ FRIS, Tohoku University, Sendai, Miyagi 980-8578, Japan \\ ${ }^{6}$ Department of Physics, Tohoku University, Sendai, Miyagi 980-8578, Japan
}

(Received 25 November 2020; accepted 30 March 2021; published 20 May 2021)

\begin{abstract}
We present a leptogenesis mechanism based on the standard type-I seesaw model that successfully operates at right-handed-neutrino masses as low as a few hundred TeV. This mechanism, which we dub wash-in leptogenesis, does not require any $C P$ violation in the neutrino sector and can be implemented even in the regime of strong wash-out. The key idea behind wash-in leptogenesis is to generalize standard freeze-out leptogenesis to a nonminimal cosmological background in which the chemical potentials of all particles not in chemical equilibrium at the temperature of leptogenesis are allowed to take arbitrary values. This sets the stage for building a plethora of new baryogenesis models where chemical potentials generated at high temperatures are reprocessed to generate a nonvanishing $B-L$ asymmetry at low temperatures. As concrete examples, we discuss wash-in leptogenesis after axion inflation and in the context of grand unification.
\end{abstract}

DOI: $10.1103 /$ PhysRevLett.126.201802

Introduction.-The cosmic imbalance between matter and antimatter [1,2] represents clear evidence for new physics beyond the standard model (SM). Early attempts to explain the baryon asymmetry of the Universe (BAU) related its origin to the $C P$-violating decays of heavy GUT particles in grand unified theories (GUTs) [3-7]. It was, however, soon realized that electroweak sphaleron processes [8] spoil this explanation. In the early Universe, sphalerons nonperturbatively wash out the baryonplus-lepton number $B+L$, which is exactly the linear combination of charges generated during standard GUT baryogenesis. This observation subsequently led to the proposal of leptogenesis [9], which links the BAU to neutrino physics in the type-I seesaw extension of the SM [10-14] and which exploits the fact that sphalerons do not violate the baryon-minus-lepton number $B-L$. Indeed, during leptogenesis, the $C P$-violating decays of righthanded neutrinos (RHNs) $N_{I}(I=1,2, \ldots)$ first create a lepton asymmetry (and, hence, nonzero $B-L$ ), which is then converted by the SM interactions in the thermal bath, including sphalerons, to a baryon asymmetry.

Standard thermal leptogenesis requires very large RHN masses, $M_{I} \gtrsim 10^{9} \mathrm{GeV}$, in order to achieve sufficient $C P$

Published by the American Physical Society under the terms of the Creative Commons Attribution 4.0 International license. Further distribution of this work must maintain attribution to the author(s) and the published article's title, journal citation, and DOI. Funded by SCOAP ${ }^{3}$. violation during RHN freeze-out $[15,16]$. This makes it hard to directly probe the RHN sector in experiments and leads to large radiative corrections to the mass of the SM Higgs boson, which aggravates the SM hierarchy problem for RHN masses above the Vissani bound $M_{I} \lesssim 10^{7} \mathrm{GeV}$ $[17,18]$. In addition, standard leptogenesis is vulnerable to strong asymmetry wash-out, if the RHN Yukawa interactions with the SM lepton-Higgs pairs $\ell_{\alpha} \phi$ are too strong [19-22].

In this Letter, we will present a mechanism to generate nonzero $B-L$ charge in the type-I seesaw model that avoids most of these shortcomings; for alternative routes to low-scale leptogenesis, see [23-30]. The key idea behind our proposal is to generalize standard freeze-out leptogenesis to a nonminimal cosmological background in which all conserved charges $C$ at the time of leptogenesis (see Table I) are allowed to take arbitrary values. In such a background, the lepton-number-violating (LNV) RHN interactions then result in a new equilibrium attractor for the chemical potentials in the plasma that generically features nonzero $B-L$, even if $B-L=0$ initially. The RHN interactions also actively drive the plasma toward this new attractor solution, which is why we dub our mechanism wash-in leptogenesis.

As we will show, wash-in leptogenesis can successfully operate down to RHN masses of a few hundred $\mathrm{TeV}$, i.e., masses shortly above the equilibration temperature of the electron Yukawa interaction [31]. The mechanism, therefore, allows one to satisfy the Vissani bound; in particular, it 
TABLE I. Decoupling of SM interactions and associated conserved charges $q_{C}$. Yukawa interactions are denoted by $y_{i}$ and weak (strong) sphalerons by WS (SS). The $\checkmark$ symbol marks efficient interactions. Hypercharge and the $\Delta_{\alpha}$ asymmetries are always preserved in the SM.

\begin{tabular}{|c|c|c|c|c|c|c|c|c|c|c|c|c|c|}
\hline & $T[\mathrm{GeV}]$ & $y_{e}$ & $y_{d s}$ & $y_{d}$ & $y_{s}$ & $y_{s b}$ & $y_{\mu}$ & $y_{c}$ & $y_{\tau}$ & $y_{b}$ & WS & SS & $y_{t}$ \\
\hline (v) & $\left(10^{5}, 10^{6}\right)$ & $q_{e}$ & $\checkmark$ & $\checkmark$ & $\checkmark$ & $\checkmark$ & $\checkmark$ & $\checkmark$ & $\checkmark$ & $\checkmark$ & $\checkmark$ & $\checkmark$ & $\checkmark$ \\
\hline (iv) & $\left(10^{6}, 10^{9}\right)$ & $q_{e}$ & $q_{2 B_{1}-B_{2}-B_{3}}$ & $q_{u-d}$ & $\checkmark$ & $\checkmark$ & $\checkmark$ & $\checkmark$ & $\checkmark$ & $\checkmark$ & $\checkmark$ & $\checkmark$ & $\checkmark$ \\
\hline (iii) & $\left(10^{9}, 10^{11-12}\right)$ & $q_{e}$ & $q_{2 B_{1}-B_{2}-B_{3}}$ & $q_{u-d}$ & $q_{d-s}$ & $q_{B_{1}-B_{2}}$ & $q_{\mu}$ & $\checkmark$ & $\checkmark$ & $\checkmark$ & $\checkmark$ & $\checkmark$ & $\checkmark$ \\
\hline (ii) & $\left(10^{11-12}, 10^{13}\right)$ & $q_{e}$ & $q_{2 B_{1}-B_{2}-B_{3}}$ & $q_{u-d}$ & $q_{d-s}$ & $q_{B_{1}-B_{2}}$ & $q_{\mu}$ & $q_{u-c}$ & $q_{\tau}$ & $q_{d-b}$ & $q_{B}$ & $\checkmark$ & $\checkmark$ \\
\hline (i) & $\left(10^{13}, 10^{15}\right)$ & $q_{e}$ & $q_{2 B_{1}-B_{2}-B_{3}}$ & $q_{u-d}$ & $q_{d-s}$ & $q_{B_{1}-B_{2}}$ & $q_{\mu}$ & $q_{u-c}$ & $q_{\tau}$ & $q_{d-b}$ & $q_{B}$ & $q_{u}$ & $\checkmark$ \\
\hline
\end{tabular}

is compatible with the neutrino option, which denotes the idea that RHNs with masses of a few $\mathrm{PeV}$ are responsible for radiatively generating the electroweak scale in the SM [32-36]. Wash-in leptogenesis is also independent of the amount of $C P$ violation in the RHN sector, which liberates it from the Davidson-Ibarra bound $M_{I} \gtrsim 10^{9} \mathrm{GeV}$; and its success is not jeopardized by large Yukawa couplings. In fact, in the presence of additional conserved charges, strong asymmetry wash-out turns into efficient asymmetry wash-in.

Our proposal builds on earlier work, which already partly considered some of the ideas presented here [3741] (see also [42]). The essential new elements of our analysis are the following: (i) We provide a systematic discussion spanning ten orders of magnitude in temperature, $T \in\left(10^{5}, 10^{15}\right) \mathrm{GeV}$. In doing so, we account for all possible unconstrained charges in each temperature regime, which allows us to develop a general toolkit for constructing new baryogenesis models; see our main results in Table II. (ii) We pay particular attention to flavor. That is, we allow for an arbitrary flavor composition of the primordial charge asymmetries, and we take into account charged-lepton flavor effects in our analysis of wash-in leptogenesis. This especially includes effects related to flavor coherence or decoherence. (iii) We go beyond LNV two-to-two scattering processes mediated by the dimension-5 Weinberg operator, considering also the ordinary decays and inverse decays of dynamical RHNs.

While wash-in leptogenesis can provide the basis for numerous new baryogenesis models, it does not represent a complete model by itself. It should rather be regarded as a general mechanism that describes how RHN interactions reprocess primordial charge asymmetries that were generated at higher temperatures. This includes the intriguing possibility of creating a nonvanishing $B-L$ asymmetry from $B-L$-symmetric initial conditions. But it is agnostic about the ultraviolet (UV) physics that is responsible for setting these initial conditions. This is an advantage, as it allows us to perform a model-independent analysis from a bottom-up perspective. The remainder of this Letter is therefore organized as follows: First, we will study wash-in leptogenesis in the spirit of an effective field theory that describes the evolution of its input parameters (i.e., the primordial charge asymmetries) from some high-energy matching scale down to low energies. Then, we will turn to concrete UV completions that illustrate how wash-in leptogenesis can successfully create the BAU, even if $B-L=0$ initially. Specifically, we will consider the generation of nonzero $B+L$ charge during GUT baryogenesis and axion inflation [44-47]. A lesson from these examples is that wash-in leptogenesis is able to resurrect baryogenesis scenarios that would otherwise suffer from

TABLE II. Numerical coefficients $x_{C}$ that describe the composition of $\mu_{B-L}^{\mathrm{eq}}=q_{B-L}^{\mathrm{eq}} 6 / T^{2}$ in terms of the conserved charges $\mu_{C}=$ $q_{C} 6 / T^{2}$ in different temperature regimes; see Eq. (15). The $\boldsymbol{X}$ symbol marks the absence of the corresponding $\mu_{C}$ due to an efficient SM interaction. The second column indicates the active flavors $\ell_{\alpha}$ with respect to $N_{1}$ interactions; see the discussion around Eq. (13). The last column contains $n_{\Delta_{\perp}}$, which vanishes in the case of $B-L$-symmetric initial conditions. $P$ and $P_{\tau}$ are model dependent and encode the flavor composition of the primordial $q_{e, \mu, \tau}$ asymmetries with respect to the $N_{1}$ wash-out direction [see the text for examples and Eqs. (S41) and (S56) [43]]. In this table and throughout the Letter, we assume vanishing global hypercharge, $\mu_{Y}=0$. For more details, see Supplemental Material [43].

\begin{tabular}{|c|c|c|c|c|c|c|c|c|c|c|c|c|c|c|}
\hline & $T_{B-L}[\mathrm{GeV}]$ & Index $\alpha$ & $\mu_{e}$ & $\mu_{2 B_{1}-B_{2}-B_{3}}$ & ${ }_{3} \mu_{u-d}$ & $\mu_{d-s}$ & $\mu_{B_{1}-B_{2}}$ & $\mu_{\mu}$ & $\mu_{u-c}$ & $\mu_{\tau}$ & \multicolumn{4}{|c|}{$\mu_{d-b} \mu_{B} \mu_{u} \mu_{\Delta_{\perp}}$} \\
\hline (v) & $\left(10^{5}, 10^{6}\right)$ & $e, \mu, \tau$ & $-\frac{3}{10}$ & $x$ & $x$ & $x$ & $x$ & $x$ & $x$ & $x$ & $x$ & $x$ & $x$ & $x$ \\
\hline (iv) & $\left(10^{6}, 10^{9}\right)$ & $e, \mu, \tau$ & $-\frac{3}{17}$ & 0 & $-\frac{7}{17}$ & $x$ & $x$ & $x$ & $x$ & $x$ & $x$ & $x$ & $x$ & $x$ \\
\hline (iii) & $\left(10^{9}, 10^{11-12}\right)$ & $\|_{\tau}, \tau$ & $\left(142-225 P_{\tau}\right) / 247$ & 0 & $-\frac{123}{247}$ & $-\frac{82}{247}$ & $\frac{123}{494}$ & $\left(142-225 P_{\tau}\right) / 247$ & $x$ & $x$ & $x$ & $x$ & $x$ & $\frac{225}{247}$ \\
\hline (ii) & $\left(10^{11-12}, 10^{13}\right)$ & $\|$ & $(-23 P+7) / 30$ & $\frac{1}{5}$ & $-\frac{3}{5}$ & $-\frac{1}{6}$ & $\begin{array}{r}494 \\
-\frac{3}{10}\end{array}$ & $(-23 P+7) / 30$ & $\frac{3}{10}$ & $(-23 P+7) / 30$ & $-\frac{4}{15}$ & $\frac{23}{90}$ & $x$ & $\frac{23}{30}$ \\
\hline (i) & $\left(10^{13}, 10^{15}\right)$ & $\|$ & $(-3 P+1) / 4$ & $\begin{array}{l}3 \\
\frac{1}{6}\end{array}$ & $-\frac{5}{6}$ & $-\frac{1}{4}$ & $-\frac{1}{4}$ & $(-3 P+1) / 4$ & $\frac{1}{4}$ & $(-3 P+1) / 4$ & $-\frac{1}{3}$ & $\frac{1}{6}$ & $\frac{1}{3}$ & $\frac{3}{4}$ \\
\hline
\end{tabular}


strong asymmetry wash-out, in a way that is more complex than simply resorting to standard leptogenesis.

Wash-in leptogenesis.-We begin by considering a particularly interesting and simple scenario: $N_{1}$-dominated wash-in leptogenesis at temperatures of a few hundred $\mathrm{TeV}$. In this temperature regime, all SM interactions are equilibrated-except for the electron Yukawa interaction, which renders the comoving charge asymmetry of right-handed electrons a classically conserved quantity, $q_{e} / s=$ const, with entropy density $s$. Its anomalous violation via the chiral plasma instability is negligibly slow for the $q_{e} / s$ values of interest [48-50]. At the same time, all chargedlepton flavors $\alpha=e, \mu, \tau$ are fully decohered, which allows us to work with the standard Boltzmann equations for the three lepton flavor asymmetries $\Delta_{\alpha}=B / 3-L_{\alpha}$ in the type-I seesaw model $[24,26]$ :

$-\left(\partial_{t}+3 H\right) q_{\Delta_{\alpha}}=\varepsilon_{1 \alpha} \Gamma_{1}\left(n_{N_{1}}-n_{N_{1}}^{\mathrm{eq}}\right)-\sum_{\beta} \gamma_{\alpha \beta}^{w} \frac{\mu_{\ell_{\beta}}+\mu_{\phi}}{T}$,

which is valid in the nonrelativistic regime $T \lesssim M_{1}$, where any $N_{1}$ chemical potential is clearly negligible because of the $N_{1}$ Majorana mass $\mu_{N_{1}} \simeq 0$. The negative sign on the left-hand side follows from $\Delta_{\alpha} \supset-L_{\alpha}$. The charge asymmetry $q_{i}$ for a particle species $i$ is defined as the difference of its particle and antiparticle number densities, $q_{i}=n_{i}-n_{\bar{l}}=g_{i} \mu_{i} T^{2} / 6$, with chemical potential $\mu_{i}$ and multiplicity $g_{i}$, while $q_{C}=\mu_{C} T^{2} / 6$ for all conserved charges $C$, with $\mu_{C}$ in Eq. (5). The first term on the right-hand side in Eq. (1) is the standard source term describing the asymmetry production from RHN decays, while the second term is the standard wash-out term, with total wash-out rate per unit volume

$\gamma_{\alpha \beta}^{w}=\gamma_{\alpha \beta}^{\mathrm{id}}+\sum_{\sigma}\left[\left(\delta_{\alpha \beta}+\delta_{\sigma \beta}\right) \gamma_{\alpha \sigma}^{\Delta L=2}+\left(\delta_{\alpha \beta}-\delta_{\sigma \beta}\right) \gamma_{\alpha \sigma}^{\Delta L=0}\right]$,

which encompasses RHN inverse decays $\gamma_{\alpha \beta}^{\text {id }}=\gamma_{1 \alpha} \delta_{\alpha \beta}$ as well as $\Delta L=2$ and lepton-flavor-violating $\Delta L=0$ twoto-two scattering processes (see [24,26] for more details).

Before we are able to solve the coupled system of equations in Eq. (1), we have to specify the relation among the chemical potentials $\mu_{\ell_{\alpha}}, \mu_{\phi}$, and $\mu_{\Delta_{\alpha}}$. In standard leptogenesis, this relation is encoded in the flavor coupling matrix $(\underline{\boldsymbol{C}})_{\alpha \beta}=C_{\alpha \beta}$ [51-56], whose structure is determined by SM spectator processes [57-59]. The crucial difference between standard leptogenesis and our scenario is that, in a nontrivial chemical background, the standard linear relation $\mu_{\ell_{\alpha}}+\mu_{\phi}=-\sum_{\beta} C_{\alpha \beta} \mu_{\Delta_{\beta}}$ turns into an affine relation:

$$
\mu_{\ell_{\alpha}}+\mu_{\phi}=\mu_{\alpha}^{0}-\sum_{\beta} C_{\alpha \beta} \mu_{\Delta_{\beta}},
$$

where, at temperatures of a few hundred $\mathrm{TeV}$, the translation by the constant shift vector $\mu_{\alpha}^{0}$ is solely induced by the conserved chemical potential of the right-handed electrons:

$$
\left(\begin{array}{l}
\mu_{\ell_{e}}+\mu_{\phi} \\
\mu_{\ell_{\mu}}+\mu_{\phi} \\
\mu_{\ell_{\tau}}+\mu_{\phi}
\end{array}\right)=\left(\begin{array}{c}
-\frac{5}{13} \\
\frac{4}{37} \\
\frac{4}{37}
\end{array}\right) \mu_{e}-\left(\begin{array}{ccc}
\frac{6}{13} & 0 & 0 \\
0 & \frac{41}{111} & \frac{4}{111} \\
0 & \frac{4}{111} & \frac{41}{111}
\end{array}\right)\left(\begin{array}{l}
\mu_{\Delta_{e}} \\
\mu_{\Delta_{\mu}} \\
\mu_{\Delta_{\tau}}
\end{array}\right) .
$$

Equations (3) and (4) follow from analyzing all $16 \mathrm{SM}$ chemical potentials $\mu_{i}\left(i=e, \mu, \tau, \ell_{e}, \ell_{\mu}, \ell_{\tau}, u, c, t, d, s, b\right.$, $\left.Q_{1}, Q_{2}, Q_{3}, \phi\right)$ : In any given temperature regime, the number of linearly independent conserved charges $C$ and the number of SM interactions in equilibrium always add up to 16; see Table I. This results in 16 constraint equations in each temperature regime that allow one to express the chemical potentials $\mu_{i}$ of all SM species as linear combinations of the conserved chemical potentials $\mu_{C}$ $\left(C=\Delta_{\alpha}, \ldots\right)$. In general, we therefore obtain a constant shift vector $\mu_{\alpha}^{0}$ in Eq. (3) of the form

$$
\mu_{\alpha}^{0}=\sum_{C \neq \Delta_{\alpha}} S_{\alpha C} \mu_{C}, \quad \mu_{C}=\sum_{i} n_{i}^{C} g_{i} \mu_{i},
$$

with charge vectors $n_{i}^{C}$ and multiplicities $g_{i}$; see [60] for details. We provide explicit expressions for $n_{i}^{C}, g_{i}$, the flavor coupling matrices $C_{\alpha \beta}$, and source matrices $S_{\alpha C}$ in all temperature regimes of interest in Supplemental Material [43].

Equations (1) and (3) tell us that the Boltzmann equations are linear in the lepton flavor asymmetries $\Delta_{\alpha}$. This allows us to split $q_{\Delta_{\alpha}}$ into contributions from thermal and wash-in leptogenesis, respectively, $q_{\Delta_{\alpha}}=q_{\Delta_{\alpha}}^{\text {th }}+q_{\Delta_{\alpha}}^{\text {win }}$, such that

$$
\left(\partial_{t}+3 H\right) q_{\Delta_{\alpha}}^{\mathrm{win}}=\sum_{\beta} \Gamma_{\alpha \beta}^{w}\left(q_{\beta}^{0}-\sum_{\sigma} C_{\beta \sigma} q_{\Delta_{\sigma}}^{\mathrm{win}}\right),
$$

where $\Gamma_{\alpha \beta}^{w}=6 / T^{3} \gamma_{\alpha \beta}^{w}$. Equation (6) is reminiscent of spontaneous baryogenesis $[61,62]$, specifically, spontaneous leptogenesis $[63,64]$, where the rolling of a (pseudo) scalar field $\varphi$ induces effective chemical potentials $\mu_{\alpha}^{0} \propto q_{\alpha}^{0}$ [60] (see also [65,66]). The difference between spontaneous leptogenesis and our scenario is that we assume nonzero primordial asymmetries stored in a set of conserved charges, whereas spontaneous leptogenesis involves time-dependent asymmetries - controlled by the interaction Lagrangian of the field $\varphi$ and not necessarily related to conserved charges - that are present only when $\varphi$ is in motion. This requires that LNV processes must be efficient exactly at the time when $\varphi$ is rolling. In our scenario, such a temporal coincidence is not needed. Still, it is 
straightforward to generalize the following analysis to timedependent charges $q_{\alpha}^{0}$ [67].

At any given temperature, the total wash-out rate is typically dominated by a single process, such that it factorizes into $\Gamma_{\alpha \beta}^{w}=P_{\alpha \beta} \Gamma_{w}$, where the temperature dependence is contained in the flavor-blind wash-out rate $\Gamma_{w}$ and where the matrix $(\underline{\boldsymbol{P}})_{\alpha \beta}=P_{\alpha \beta}$ encodes the flavor structure. In this case, it is then possible to write down an exact solution of Eq. (6). For arbitrary initial conditions $q_{\Delta_{\beta}}^{\text {ini }}$, we find

$$
q_{\Delta_{\alpha}}^{\mathrm{win}}=\sum_{\beta}\left(\delta_{\alpha \beta}-E_{\alpha \beta}\right) q_{\Delta_{\beta}}^{\mathrm{eq}}+\sum_{\beta} E_{\alpha \beta} q_{\Delta_{\beta}}^{\mathrm{ini}} \frac{s}{s^{\mathrm{ini}}} .
$$

$q_{\Delta_{\alpha}}^{\mathrm{eq}}$ is the equilibrium attractor in the presence of RHNs,

$$
q_{\Delta_{\alpha}}^{\mathrm{eq}}=\sum_{\beta} C_{\alpha \beta}^{-1} q_{\beta}^{0}=\sum_{\beta} \sum_{C \neq \Delta_{\alpha}} C_{\alpha \beta}^{-1} S_{\beta C} q_{C}
$$

which can also be derived from Eq. (3) by requiring all RHN interactions to be in equilibrium, $\mu_{\ell_{\alpha}}+\mu_{\phi}=$ $\mu_{N_{1}}=0$. The matrix $(\underline{\boldsymbol{E}})_{\alpha \beta}=E_{\alpha \beta}$ describes how the RHN interactions actively drive the plasma exponentially close to this solution:

$\underline{\boldsymbol{E}}=\exp \left(-w K_{1} \underline{\boldsymbol{P C}}\right), \quad w=\frac{1}{K_{1}} \int_{0}^{\infty} d z \frac{\Gamma_{w}}{z H}, \quad z=\frac{M_{1}}{T}$,

where $K_{1}$ denotes the standard $N_{1}$ decay parameter:

$$
K_{1}=\frac{\Gamma_{1}(T=0)}{H\left(T=M_{1}\right)} .
$$

At temperatures of a few hundred $\mathrm{TeV}$, the total wash-out rate is dominated by inverse decays, such that $P_{\alpha \beta}=$ $p_{1 \alpha} \delta_{\alpha \beta}$ and

$\underline{\boldsymbol{E}}=\exp \left(-w K_{1} \underline{\boldsymbol{C}}_{1}\right), \quad\left(\underline{\boldsymbol{C}}_{1}\right)_{\alpha \beta}=p_{1 \alpha} C_{\alpha \beta}, \quad p_{1 \alpha}=\frac{\Gamma_{1 \alpha}}{\Gamma_{1}}$,

where $w \approx 3 \pi / 4$ assuming Maxwell-Boltzmann statistics for all particles [68]. For strong wash-in, $K_{1} \gg 1$, and a generic flavor structure, $p_{1 \alpha} k<1$, all entries of $\underline{\boldsymbol{E}}$ are exponentially suppressed. The total washed-in $B-L$ asymmetry then reads

$$
q_{B-L}^{\mathrm{win}} \simeq q_{B-L}^{\mathrm{eq}}=\sum_{\alpha} q_{\Delta_{\alpha}}^{\mathrm{eq}}=-\frac{3}{10} q_{e}
$$

which also immediately follows from Eq. (4). Any UV mechanism that results in $q_{e} \neq 0$ at high temperatures, thus, induces nonzero $B-L$ at temperatures of a few hundred $\mathrm{TeV}$.
Flavor effects.-Next, let us generalize the above discussion to arbitrary temperatures $T \in\left(10^{5}, 10^{15}\right) \mathrm{GeV}$. Equations (1)-(9), except for Eq. (4), remain valid in this case, the only difference being that the meaning of the flavor index $\alpha$ is now different. At $T \in\left(10^{9}, 10^{11-12}\right) \mathrm{GeV}$, electrons and muons propagate as coherent states, which means $\alpha=\|_{\tau}, \tau$, while at temperatures $T \in$ $\left(10^{11-12}, 10^{15}\right) \mathrm{GeV}$, all three charged leptons propagate in coherent superpositions, such that $\alpha=\|$. Here, $\ell_{\|}$ represents the coherent single-flavor field that can be created and destroyed by $N_{1}$ interactions, and $\ell_{\|_{\tau}}$ is the same field after projecting out its $\tau$ component. Denoting the $N_{1}$ Yukawa couplings by $h_{1}^{e}, h_{1}^{\mu}$, and $h_{1}^{\tau}$, we can write

$h_{\|} e_{\|}=h_{1}^{e} \ell_{e}+h_{1}^{\mu} \ell_{\mu}+h_{1}^{\tau} \ell_{\tau}, \quad h_{\|_{\tau}} \ell_{\|_{\tau}}=h_{1}^{e} \ell_{e}+h_{1}^{\mu} \ell_{\mu}$,

where $h_{\|}^{2}=\left|h_{1}^{e}\right|^{2}+\left|h_{1}^{\mu}\right|^{2}+\left|h_{1}^{\tau}\right|^{2}$ and $h_{\|_{\tau}}^{2}=\left|h_{1}^{e}\right|^{2}+\left|h_{1}^{\mu}\right|^{2}$. Flavor coherence at higher temperatures also implies that some flavor asymmetry $\Delta_{\perp}$ can escape wash-in leptogenesis:

$\Delta_{\perp}= \begin{cases}B / 3-L_{\perp}, & T \in\left(10^{9}, 10^{11-12}\right) \mathrm{GeV}, \\ 2 B / 3-L_{\perp_{1}}-L_{\perp_{2}}, & T \in\left(10^{11-12}, 10^{15}\right) \mathrm{GeV},\end{cases}$

where $\ell_{\perp}$ is perpendicular to $\ell_{\tau}$ and $\ell_{\|_{\tau}}$ and where $\ell_{\perp_{1}}$ and $\ell_{\perp_{2}}$ span the two-dimensional flavor space perpendicular to $\ell_{\|}$. Making use of these definitions and assuming again strong wash-in and generic RHN couplings, Eq. (12) now turns into

$q_{B-L}^{\mathrm{eq}}=\sum_{C \neq \Delta_{\alpha}} x_{C} q_{C}, \quad x_{C}=\delta_{C \Delta_{\perp}}+\sum_{\alpha, \beta} C_{\alpha \beta}^{-1} S_{\beta C}$,

where the numerical coefficients $x_{C}$ are listed in Table II. This asymmetry remains conserved as soon as the RHN interactions become inefficient at some high temperature $T_{B-L}$ [22]. We therefore obtain for the present-day BAU

$$
\left.\frac{q_{B}}{s}\right|_{\text {today }}=\left.c_{\mathrm{sph}} \frac{q_{B-L}^{\mathrm{th}}+q_{B-L}^{\mathrm{win}}}{s}\right|_{T_{B-L}},
$$

where $c_{\mathrm{sph}} \simeq 12 / 37$ [69]. Note that the standard contribution from thermal leptogenesis may be suppressed because of strong wash-out or insufficient $C P$ violation.

Equation (15) and Table II are our main results, which serve as a general toolkit to construct new baryogenesis models by implementing the following algorithm: (i) Conceive a UV model that leads to primordial chemical potentials $\mu_{i}$ for some particle species $i$. (ii) Determine the corresponding conserved charges $\mu_{C}$. (iii) Specify the $N_{1}$ mass and, hence, relevant temperature scale for 
leptogenesis, $T_{B-L}$. (iv) Compute the final BAU according to Eqs. (15) and (16).

Possible UV completions. - Let us now showcase two possibilities for generating primordial charge asymmetries prior to wash-in leptogenesis. Both scenarios result in $B+L \neq 0$ but preserve $B-L$. First, we consider $\mathrm{SU}(5)$ unification, where the decay of the heavy colored Higgs field $H^{c} \subset \mathbf{5}$ mainly proceeds via the third-generation Yukawa coupling, $H^{c} \rightarrow \bar{Q}_{3} \bar{Q}_{3}, t \tau, Q_{3} \ell_{\tau}, \bar{t} \bar{b}[7,70,71]$. The production and decay of $H^{c}$ bosons after inflation in the SU(5)-broken phase (see, e.g., Refs. [72,73] for a viable scenario) then results in $\mu_{Q_{3}}=\mu_{\ell_{\tau}}=-\mu_{\tau}=\mu_{0}$, $\mu_{t}=-2 \mu_{0} / 3$, and $\mu_{b}=-\mu_{0} / 3$ or, equivalently, $\mu_{B}=-\mu_{2 B_{1}-B_{2}-B_{3}}=\mu_{\ell_{\tau}}=-\mu_{\tau}=3 \mu_{d-b}=\mu_{0}$, while all other chemical potentials vanish. Here, $\mu_{0}$ is determined by the decay rate, $C P$ violation, and production mechanism of the colored Higgs field. This scenario sets the stage for wash-in leptogenesis above the equilibration temperature of the tau Yukawa interaction, $T \gtrsim 10^{11-12} \mathrm{GeV}$. Similarly, one can construct models where extra Higgs scalars also generate primordial asymmetries in the first two fermion generations. The initial $q_{e, \mu, \tau}$ asymmetries are then encoded in general fields $\bar{e}=c_{e} e+c_{\mu} \mu+c_{\tau} \tau$ or $\bar{e}_{\tau}=c_{e}^{\tau} e+c_{\mu}^{\tau} \mu$, such that

$P=\left|a_{e} c_{e}^{*}+a_{\mu} c_{\mu}^{*}+a_{\tau} c_{\tau}^{*}\right|^{2}, \quad P_{\tau}=\left|b_{e} c_{e}^{\tau *}+b_{\mu} c_{\mu}^{\tau *}\right|^{2}$

in Table II, where $a_{e, \mu, \tau}=h_{1}^{e, \mu, \tau} / h_{\|}$and $b_{e, \mu}=h_{1}^{e, \mu} / h_{\|_{\tau}}$.

Our second example is axion inflation featuring a coupling of the axion-inflaton field $\varphi$ to the Chern-Simons term of the hypercharge gauge field, $\varphi /(4 \Lambda) Y_{\mu \nu} \tilde{Y}^{\mu \nu}$ [74]. This coupling sources nonvanishing $\left\langle Y_{\mu \nu} \tilde{Y}^{\mu \nu}\right\rangle$ during inflation [75-77], which induces primordial chemical potentials for all SM fermion species via the SM chiral anomaly [78,79], $\mu_{i} / T= \pm 3\left(n_{i}^{Y}\right)^{2} \alpha_{Y} / \pi\left(h_{Y} / T^{3}\right)_{\text {rh }}$ [46,47], with hypercharge fine-structure constant $\alpha_{Y}$, hypercharges $n_{i}^{Y}$, and \pm for leftand right-handed fermions. $h_{Y}=\left\langle\boldsymbol{A}_{Y} \cdot \boldsymbol{B}_{Y}\right\rangle / a^{3}$ is the physical hypermagnetic helicity density, which is defined in terms of the comoving vector potential $\boldsymbol{A}_{Y}$, comoving flux density $\boldsymbol{B}_{Y}$, and cosmic scale factor $a$. In the parameter region where $h_{Y} / T^{3}$ is approximately conserved [47,80-82], its value at reheating after inflation dictates the magnitude of the conserved charges in each temperature regime. For $T \in\left(10^{5}, 10^{6}\right) \mathrm{GeV}$, e.g., we have $\mu_{e} / T=$ $-3 \alpha_{Y} / \pi\left(h_{Y} / T^{3}\right)_{\mathrm{rh}}$ and, hence, $\mu_{B-L} / T=9 / 10 \alpha_{Y} /$ $\pi\left(h_{Y} / T^{3}\right)_{\text {rh }}$. Axion inflation with a Hubble rate of $H_{\text {inf }} \sim 10^{10} \mathrm{GeV}$ can therefore readily give rise to the observed baryon asymmetry [47]. The evolution of $B$ and $L$ in this scenario is schematically shown in Fig. 1. Axion inflation produces all lepton flavors in a symmetric way, meaning $P=1 / 3$ and $P_{\tau}=1 / 2$ in Table II.

Conclusions. - In this Letter, we presented a systematic discussion of wash-in leptogenesis, a mechanism to generate nonzero $B-L$ in the type-I seesaw model. Our mechanism successfully operates at low RHN masses,

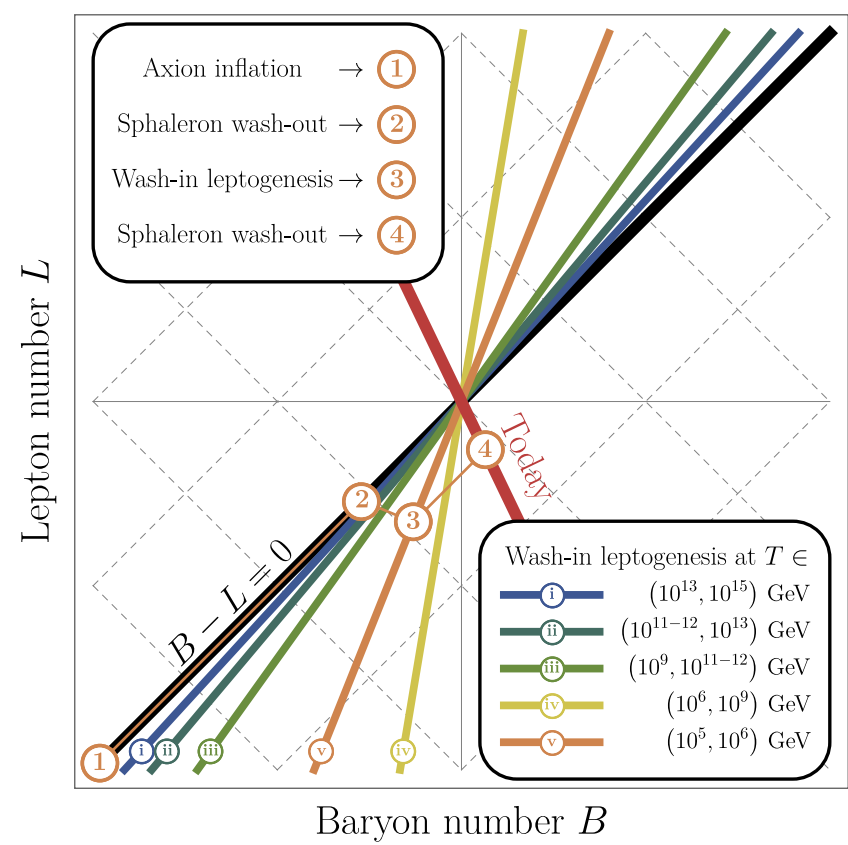

FIG. 1. Schematic evolution of $B$ and $L$ in arbitrary units after axion inflation. The colorful straight lines represent the equilibrium attractors of wash-in leptogenesis in different temperature regimes.

strong wash-out, negligible $C P$ violation in RHN decays, and $B-L$-symmetric initial conditions. We focused on $N_{1^{-}}$ dominated wash-in leptogenesis; however, the inclusion of heavy-neutrino flavor effects [83], or even the generalization to a density-matrix formalism [84-86], is straightforward. Similarly, one may generalize our mechanism to other sources of LNV in the early Universe. The general concept of wash-in leptogenesis opens the door to a plethora of possibilities.

We thank Apostolos Pilaftsis, Mikhail Shaposhnikov, and Daniele Teresi for helpful comments. K. K. was supported by JSPS KAKENHI, Grant-in-Aid for Scientific Research No. JP19K03842 and Grant-in-Aid for Scientific Research on Innovative Areas No. 19H04610. K. M. was supported by Deutsche Forschungsgemeinschaft under Germany's Excellence Strategy-EXC 2121 Quantum Universe390833306. M. Y. was supported by the Leading Initiative for Excellent Young Researchers, Ministry of Education, Culture, Sports, Science and Technology (MEXT), Japan, and by JSPS KAKENHI Grants No. 20H05851, No. 21K13910, and No. JP20K22344. This project has received funding from the European Union's Horizon 2020 Research and Innovation Program under Grant Agreement No. 796961, "AxiBAU” (K. S.).

\footnotetext{
*valerie.domcke@cern.ch

†kohei.kamada@resceu.s.u-tokyo.ac.jp

†kyohei.mukaida@cern.ch
} 
$\S$ kai.schmitz@cern.ch

"m.yamada@tohoku.ac.jp

[1] Planck Collaboration, Planck 2018 results. VI. Cosmological parameters, Astron. Astrophys. 641, A6 (2020).

[2] Particle Data Group, Review of particle physics, Prog. Theor. Exp. Phys. 2020, 083C01 (2020).

[3] M. Yoshimura, Unified Gauge Theories and the Baryon Number of the Universe, Phys. Rev. Lett. 41, 281 (1978).

[4] S. Dimopoulos and L. Susskind, On the baryon number of the Universe, Phys. Rev. D 18, 4500 (1978).

[5] D. Toussaint, S. B. Treiman, F. Wilczek, and A. Zee, Matterantimatter accounting, thermodynamics, and black hole radiation, Phys. Rev. D 19, 1036 (1979).

[6] S. Weinberg, Cosmological Production of Baryons, Phys. Rev. Lett. 42, 850 (1979).

[7] S. M. Barr, G. Segre, and H. Weldon, The magnitude of the cosmological baryon asymmetry, Phys. Rev. D 20, 2494 (1979).

[8] V. Kuzmin, V. Rubakov, and M. Shaposhnikov, On the anomalous electroweak baryon number nonconservation in the early universe, Phys. Lett. 155B, 36 (1985).

[9] M. Fukugita and T. Yanagida, Baryogenesis without grand unification, Phys. Lett. B 174, 45 (1986).

[10] P. Minkowski, $\mu \rightarrow e \gamma$ at a rate of one out of $10^{9}$ muon decays?, Phys. Lett. 67B, 421 (1977).

[11] T. Yanagida, Horizontal gauge symmetry and masses of neutrinos, Conf. Proc. C 7902131, 261 (1979).

[12] T. Yanagida, Horizontal symmetry and masses of neutrinos, Prog. Theor. Phys. 64, 1103 (1980).

[13] M. Gell-Mann, P. Ramond, and R. Slansky, Complex spinors and unified theories, arXiv:1306.4669.

[14] R. N. Mohapatra and G. Senjanovic, Neutrino Mass and Spontaneous Parity Nonconservation, Phys. Rev. Lett. 44, 912 (1980).

[15] S. Davidson and A. Ibarra, A lower bound on the righthanded neutrino mass from leptogenesis, Phys. Lett. B 535, 25 (2002).

[16] W. Buchmuller, P. Di Bari, and M. Plumacher, Cosmic microwave background, matter-antimatter asymmetry and neutrino masses, Nucl. Phys. B643, 367 (2002).

[17] F. Vissani, Do experiments suggest a hierarchy problem?, Phys. Rev. D 57, 7027 (1998).

[18] J. D. Clarke, R. Foot, and R. R. Volkas, Electroweak naturalness in the three-flavor type I seesaw model and implications for leptogenesis, Phys. Rev. D 91, 073009 (2015).

[19] W. Buchmuller, P. Di Bari, and M. Plumacher, A bound on neutrino masses from baryogenesis, Phys. Lett. B 547, 128 (2002).

[20] W. Buchmuller, P. Di Bari, and M. Plumacher, The neutrino mass window for baryogenesis, Nucl. Phys. B665, 445 (2003).

[21] G. Giudice, A. Notari, M. Raidal, A. Riotto, and A. Strumia, Towards a complete theory of thermal leptogenesis in the SM and MSSM, Nucl. Phys. B685, 89 (2004).

[22] W. Buchmuller, P. Di Bari, and M. Plumacher, Leptogenesis for pedestrians, Ann. Phys. (Amsterdam) 315, 305 (2005).

[23] E. K. Akhmedov, V. A. Rubakov, and A. Y. Smirnov, Baryogenesis via Neutrino Oscillations, Phys. Rev. Lett. 81, 1359 (1998).
[24] A. Pilaftsis and T. E. Underwood, Resonant leptogenesis, Nucl. Phys. B692, 303 (2004).

[25] T. Asaka and M. Shaposhnikov, The $\nu$ MSM, dark matter and baryon asymmetry of the universe, Phys. Lett. B 620, 17 (2005).

[26] A. Pilaftsis and T.E. J. Underwood, Electroweak-scale resonant leptogenesis, Phys. Rev. D 72, 113001 (2005).

[27] T. Hambye and D. Teresi, Higgs Doublet Decay as the Origin of the Baryon Asymmetry, Phys. Rev. Lett. 117, 091801 (2016).

[28] J. Klarić, M. Shaposhnikov, and I. Timiryasov, Uniting lowscale leptogeneses, arXiv:2008.13771.

[29] A. Granelli, K. Moffat, and S. Petcov, Flavored resonant leptogenesis at sub-TeV scales, arXiv:2009.03166.

[30] D. Bodeker and W. Buchmuller, Baryogenesis from the weak scale to the GUT scale, arXiv:2009.07294.

[31] D. Bödeker and D. Schröder, Equilibration of right-handed electrons, J. Cosmol. Astropart. Phys. 05 (2019) 010.

[32] I. Brivio and M. Trott, Radiatively Generating the Higgs Potential and Electroweak Scale via the Seesaw Mechanism, Phys. Rev. Lett. 119, 141801 (2017).

[33] I. Brivio and M. Trott, Examining the neutrino option, J. High Energy Phys. 02 (2019) 107.

[34] V. Brdar, A. J. Helmboldt, S. Iwamoto, and K. Schmitz, Type I seesaw as the common origin of neutrino mass, baryon asymmetry, and the electroweak scale, Phys. Rev. D 100, 075029 (2019).

[35] I. Brivio, K. Moffat, S. Pascoli, S. Petcov, and J. Turner, Leptogenesis in the neutrino option, J. High Energy Phys. 10 (2019) 059.

[36] I. Brivio, J. Talbert, and M. Trott, No-go limitations on UV completions of the neutrino option, Phys. Rev. D 103, 015012 (2021).

[37] B. A. Campbell, S. Davidson, J. R. Ellis, and K. A. Olive, On the baryon, lepton flavor and right-handed electron asymmetries of the universe, Phys. Lett. B 297, 118 (1992).

[38] J. M. Cline, K. Kainulainen, and K. A. Olive, On the Erasure and Regeneration of the Primordial Baryon Asymmetry by Sphalerons, Phys. Rev. Lett. 71, 2372 (1993).

[39] J. M. Cline, K. Kainulainen, and K. A. Olive, Protecting the primordial baryon asymmetry from erasure by sphalerons, Phys. Rev. D 49, 6394 (1994).

[40] M. Fukugita and T. Yanagida, Resurrection of Grand Unified Theory Baryogenesis, Phys. Rev. Lett. 89, 131602 (2002).

[41] C. S. Fong, Baryogenesis from symmetry principle, Phys. Lett. B 752, 247 (2016).

[42] K. Dick, M. Lindner, M. Ratz, and D. Wright, Leptogenesis with Dirac Neutrinos, Phys. Rev. Lett. 84, 4039 (2000).

[43] See Supplemental Material at http://link.aps.org/supplemental/ 10.1103/PhysRevLett.126.201802 for an explicit derivation of the flavor coupling matrices, source matrices, and numerical coefficients in Eq. (15) and Table. II.

[44] P. Adshead and E. I. Sfakianakis, Fermion production during and after axion inflation, J. Cosmol. Astropart. Phys. 11 (2015) 021.

[45] P. Adshead, L. Pearce, M. Peloso, M. A. Roberts, and L. Sorbo, Phenomenology of fermion production during axion inflation, J. Cosmol. Astropart. Phys. 06 (2018) 020. 
[46] V. Domcke and K. Mukaida, Gauge field and fermion production during axion inflation, J. Cosmol. Astropart. Phys. 11 (2018) 020.

[47] V. Domcke, B. von Harling, E. Morgante, and K. Mukaida, Baryogenesis from axion inflation, J. Cosmol. Astropart. Phys. 10 (2019) 032.

[48] M. Joyce and M. E. Shaposhnikov, Primordial Magnetic Fields, Right-Handed Electrons, and the Abelian Anomaly, Phys. Rev. Lett. 79, 1193 (1997).

[49] K. Kamada, Return of grand unified theory baryogenesis: Source of helical hypermagnetic fields for the baryon asymmetry of the universe, Phys. Rev. D 97, 103506 (2018).

[50] D. G. Figueroa, A. Florio, and M. Shaposhnikov, Chiral charge dynamics in Abelian gauge theories at finite temperature, J. High Energy Phys. 10 (2019) 142.

[51] R. Barbieri, P. Creminelli, A. Strumia, and N. Tetradis, Baryogenesis through leptogenesis, Nucl. Phys. B575, 61 (2000).

[52] A. Abada, S. Davidson, F.-X. Josse-Michaux, M. Losada, and A. Riotto, Flavor issues in leptogenesis, J. Cosmol. Astropart. Phys. 04 (2006) 004.

[53] E. Nardi, Y. Nir, E. Roulet, and J. Racker, The importance of flavor in leptogenesis, J. High Energy Phys. 01 (2006) 164.

[54] A. Abada, S. Davidson, A. Ibarra, F.-X. Josse-Michaux, M. Losada, and A. Riotto, Flavour matters in leptogenesis, J. High Energy Phys. 09 (2006) 010.

[55] S. Blanchet and P. Di Bari, Flavor effects on leptogenesis predictions, J. Cosmol. Astropart. Phys. 03 (2007) 018.

[56] S. Antusch, S. King, and A. Riotto, Flavour-dependent leptogenesis with sequential dominance, J. Cosmol. Astropart. Phys. 11 (2006) 011.

[57] W. Buchmuller and M. Plumacher, Spectator processes and baryogenesis, Phys. Lett. B 511, 74 (2001).

[58] B. Garbrecht and P. Schwaller, Spectator effects during leptogenesis in the strong washout regime, J. Cosmol. Astropart. Phys. 10 (2014) 012.

[59] B. Garbrecht, P. Klose, and C. Tamarit, Relativistic and spectator effects in leptogenesis with heavy sterile neutrinos, J. High Energy Phys. 02 (2020) 117.

[60] V. Domcke, Y. Ema, K. Mukaida, and M. Yamada, Spontaneous baryogenesis from axions with generic couplings, J. High Energy Phys. 08 (2020) 096.

[61] A. G. Cohen and D. B. Kaplan, Thermodynamic generation of the baryon asymmetry, Phys. Lett. B 199, 251 (1987).

[62] A. G. Cohen and D. B. Kaplan, Spontaneous baryogenesis, Nucl. Phys. B308, 913 (1988).

[63] A. Kusenko, K. Schmitz, and T. T. Yanagida, Leptogenesis via Axion Oscillations after Inflation, Phys. Rev. Lett. 115, 011302 (2015).

[64] M. Ibe and K. Kaneta, Spontaneous thermal Leptogenesis via Majoron oscillation, Phys. Rev. D 92, 035019 (2015).

[65] R. T. Co, L. J. Hall, and K. Harigaya, Predictions for axion couplings from ALP cogenesis, J. High Energy Phys. 01 (2021) 172.

[66] R. T. Co, N. Fernandez, A. Ghalsasi, L. J. Hall, and K. Harigaya, Lepto-axiogenesis, J. High Energy Phys. 03 (2021) 017.
[67] V. Domcke, K. Kamada, K. Mukaida, K. Schmitz, and M. Yamada (to be published).

[68] S. Antusch, P. Di Bari, D. A. Jones, and S. F. King, A fuller flavour treatment of $\mathrm{N}_{2}$-dominated leptogenesis, Nucl. Phys. B856, 180 (2012).

[69] M. Laine and M. E. Shaposhnikov, A remark on sphaleron erasure of baryon asymmetry, Phys. Rev. D 61, 117302 (2000).

[70] D. V. Nanopoulos and S. Weinberg, Mechanisms for cosmological baryon production, Phys. Rev. D 20, 2484 (1979).

[71] A. Yildiz and P. H. Cox, Net baryon number and $C P$ violation with unified fields, Phys. Rev. D 21, 906 (1980).

[72] E. W. Kolb, A. D. Linde, and A. Riotto, GUT Baryogenesis after Preheating, Phys. Rev. Lett. 77, 4290 (1996).

[73] E. W. Kolb, A. Riotto, and I. I. Tkachev, GUT baryogenesis after preheating: Numerical study of the production and decay of X bosons, Phys. Lett. B 423, 348 (1998).

[74] D. Jiménez, K. Kamada, K. Schmitz, and X.-J. Xu, Baryon asymmetry and gravitational waves from pseudoscalar inflation, J. Cosmol. Astropart. Phys. 12 (2017) 011.

[75] M. S. Turner and L. M. Widrow, Inflation produced, large scale magnetic fields, Phys. Rev. D 37, 2743 (1988).

[76] W. D. Garretson, G. B. Field, and S. M. Carroll, Primordial magnetic fields from pseudoGoldstone bosons, Phys. Rev. D 46, 5346 (1992).

[77] M. M. Anber and L. Sorbo, N-flationary magnetic fields, J. Cosmol. Astropart. Phys. 10 (2006) 018.

[78] S. L. Adler, Axial vector vertex in spinor electrodynamics, Phys. Rev. 177, 2426 (1969).

[79] J. Bell and R. Jackiw, A PCAC puzzle: $\pi^{0} \rightarrow \gamma \gamma$ in the $\sigma$ model, Nuovo Cimento A 60, 47 (1969).

[80] A. Pouquet, U. Frisch, and J. Leorat, Strong MHD helical turbulence and the nonlinear dynamo effect, J. Fluid Mech. 77, 321 (1976).

[81] R. Banerjee and K. Jedamzik, The evolution of cosmic magnetic fields: From the very early universe, to recombination, to the present, Phys. Rev. D 70, 123003 (2004).

[82] T. Kahniashvili, A. G. Tevzadze, A. Brandenburg, and A. Neronov, Evolution of primordial magnetic fields from phase transitions, Phys. Rev. D 87, 083007 (2013).

[83] E. Bertuzzo, P. Di Bari, and L. Marzola, The problem of the initial conditions in flavoured leptogenesis and the tauon $\mathrm{N}_{2}$-dominated scenario, Nucl. Phys. B849, 521 (2011).

[84] S. Blanchet, P. Di Bari, D. A. Jones, and L. Marzola, Leptogenesis with heavy neutrino flavours: from density matrix to Boltzmann equations, J. Cosmol. Astropart. Phys. 01 (2013) 041.

[85] P. Bhupal Dev, P. Millington, A. Pilaftsis, and D. Teresi, Flavour covariant transport equations: An application to resonant leptogenesis, Nucl. Phys. B886, 569 (2014).

[86] P. S. B. Dev, P. Millington, A. Pilaftsis, and D. Teresi, Corrigendum to "Flavour covariant transport equations: An application to resonant leptogenesis", Nucl. Phys. B897, 749 (2015). 